\title{
NEW METHOD FOR DETERMINATION OF DRAIN SATURATION VOLTAGE IN SHORT CHANNEL MOS DEVICES BETWEEN LIQUID HELIUM TO ROOM TEMPERATURE
}

\author{
Y. AMHOUCHE ${ }^{a}$, A. EL ABBASSI ${ }^{a}, K_{\text {. RAÏS }}^{a, *}$, \\ E. BENDADA ${ }^{\mathrm{b}}$ and R. RMAILY ${ }^{\mathrm{a}}$ \\ ${ }^{a}$ Laboratoire de Caractérisation des Composants à Semi-Conducteurs, \\ Faculté des Sciences Chouaib Doukkali, Departement de Physique, \\ B.P. 20, El Jadida, Maroc; ${ }^{b}$ Laboratoire de la Microélectronique et de \\ l'Instrumentation, Faculté des Sciences, B.P. 509, Errachidia, Maroc
}

(Received 15 December 2000; In final form 19 March 2001)

A new method for drain saturation voltage extraction in submicron MOSFETs is presented. It is based on measurements of the partial derivative of the impact ionization rate. The method has been tested using main of channel length MOSFET devices and compared with others methods.

Keywords: MOS transistor; Drain saturation voltage; Substrate current

\section{INTRODUCTION}

Drain saturation voltage $V d_{\text {sat }}$ is an important parameter in MOSFET modelling and process control. The existing method for $V d_{\text {sat }}$ versus the gate voltage $V g$ extraction can be divided into two categories. The first [1,2], is based on $I d-V d$ plotted curves where $I d$ is the drain current and $V d$ is the drain voltage and the second $[3,4]$, is based on the unique dependence $\left(V d-V d_{\text {sat }}\right)$ of the ratio $(I s u b / I d)$ where $I s u b$ represent the substrate current.

*Corresponding author. 
In this letter a new technique for an accurate determination of drain saturation voltage can be classed with the second methods, and give directly the continuous $V d_{\text {sat }}(V g)$ characteristics.

\section{THEORY}

Generally, according to the lucky electron model [5], since the impact ionization rate is proportional to $\exp \left[-B /\left(V d-V d_{\text {sat }}\right)\right]$, where $B$ is a constant and ( $\left.V d-V d_{\text {sat }}\right)$ is proportional to the drain field. In the literature [6], we found one of the follows expressions of the ratio Isub/Id.

$$
I s u b / I d=A \exp \left[-B /\left(V d-V d_{\text {sat }}\right)\right]
$$

or

$$
I s u b / I d=A^{\prime}\left(V d-V d_{s a t}\right) \exp \left[-B^{\prime} /\left(V d-V d_{\text {sat }}\right)\right]
$$

where $A^{\prime}, A, B^{\prime}$ and $B$ are constants. $B^{\prime}$ and $B$ have a voltage dimension and are function of the carrier mean free path, and of the length pinch-off region. We note that (1) and (2) are all dependants on the difference $\left(V d-V d_{\text {sat }}\right)$.

We pose $X(V g, V d)=V d-V d_{\text {sat }}$ and $Y(V g, V d)=I s u b / I d$, the ratio of partial derivative $\mathrm{Y}$ on $V d$ and $V g$ can be calculated as:

$$
\frac{\partial Y / \partial V d}{\partial Y / \partial V g}=-\frac{1}{d V d_{\mathrm{sat}} / d V g}
$$

The characteristic $V d_{\text {sat }}(V g)$ is also obtained by the following integral:

$$
V d_{\text {sat }}(V g)=-\int \frac{(\partial Y / \partial V g)}{(\partial Y / \partial V d)} d V g
$$

\section{RESULTS AND DISCUSSION}

The previous analysis is applied at room temperature on the $\mathrm{N}$ Channel CNET MOSFET devices, with channel width $W$ is $50 \mu \mathrm{m}$ and channel lengths are $(L=0.9,1.5$ and $10 \mu \mathrm{m})$ the Light Doped Drain 
Region (LDD) doping is $5.10^{13} / \mathrm{cm}^{3}$, the channel doping $\mathrm{Na}$ ranging between $10^{15}$ and $10^{16} / \mathrm{cm}^{3}$, the oxide thickness $t_{o x}$ is $15 \mathrm{~nm}$. The method is also compared to the Chan et al., method [3].

The Figure 1 shows $V d_{\text {sat }}(V g)$ characteristics for three different channel lengths $(0.9,1.5$ and $10 \mu \mathrm{m})$. The experimental points on these curves are obtained with Chan et al., method [3], the lines curves are obtained with our method. We can see on this figure that the Chan et al., method and our model are in good agreement. Generally, the saturation drain voltage $V d_{\text {sat }}$ decrease with decreasing channel length and increase with increasing $\mathbf{V g}$. Indeed, the increase of $\mathbf{V g}$ provokes the increase of inversion layer charge requiring an important drain voltage to ensure saturation.

The Figure 2 shows $V d_{\text {sat }}(V g)$ characteristics for five different temperatures. $V d_{\text {sat }}$ increase with temperature at weak gate voltage and becomes not more sensitive at high gate voltage. We note that the

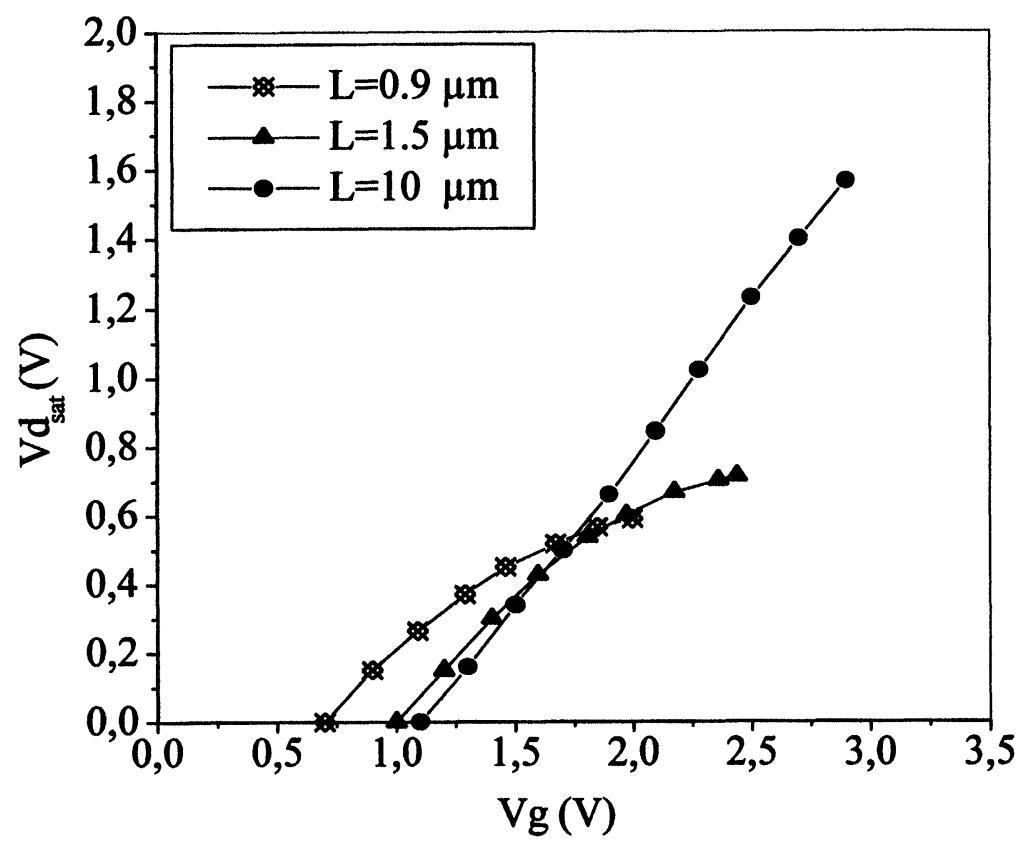

FIGURE $1 V d_{\text {sat }}$ versus $V g$ characteristics for three different Channel lengths. $(L=0.9$, 1.5 and $10 \mu \mathrm{m})$. 


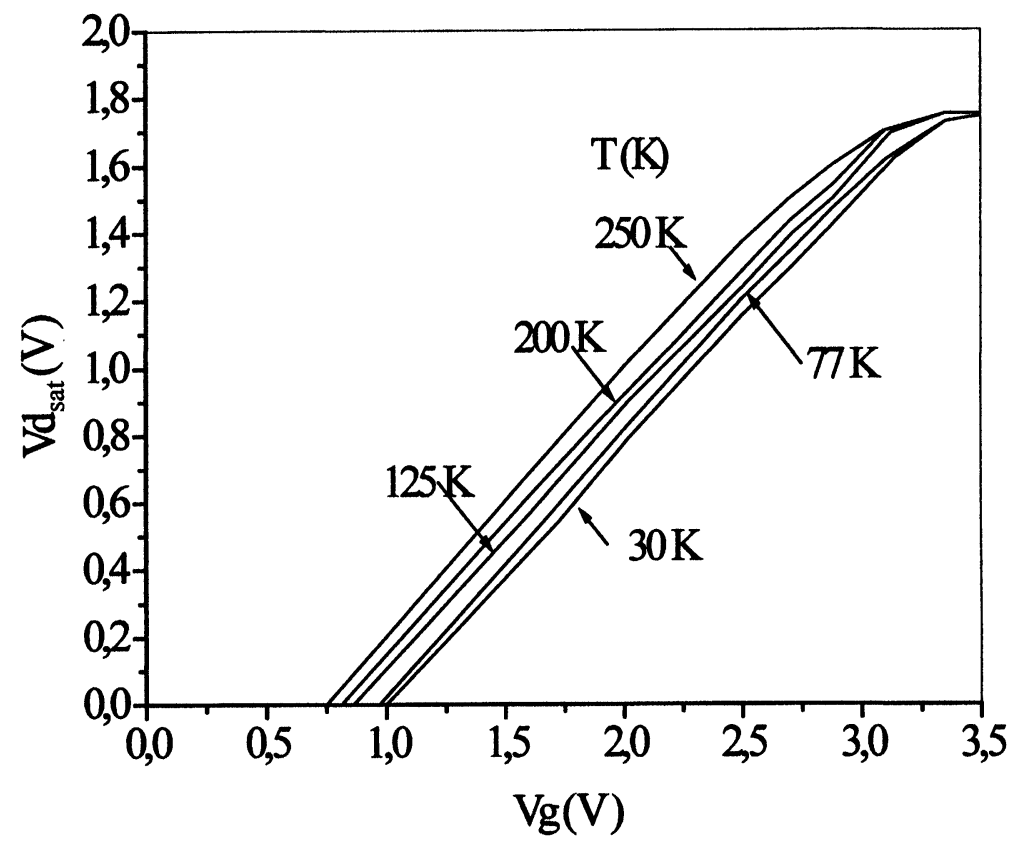

FIGURE $2 V d_{\text {sat }}$ versus $V g$ characteristics for five temperatures. (NMOS, $W / L=$ $\left.50 / 10, t_{o x}=15 \mathrm{~nm}\right)$.

expression (1) and (2) are also valid at low temperature MOS operation [6].

In order to found the method validity domain we have used $V d_{\text {sat }}(V g)$ characteristics to determine A and B parameters for $1.5 \mu \mathrm{m}$ channel length, and insure that there are constants for different potential gate. Using the expression (1), we can determine $A$ and $B$ parameters value by:

$$
B=X^{2} \partial(\log Y) / \partial V d
$$

and

$$
A=Y \exp (B / X)
$$

The plotted curves $A$ and $B$ versus $V g$, Figures 3 and 4 shows that these parameters are nearly constants in $I s u b(V g)$ apparition i.e., in strong inversion, their values are $A=8.5$ and $B=23 \mathrm{~V}$. In the other hand, these parameters are identical to the fitting parameters in the Isub/Id versus $1 /\left(V d-V d_{\text {sat }}\right)$ plots. 


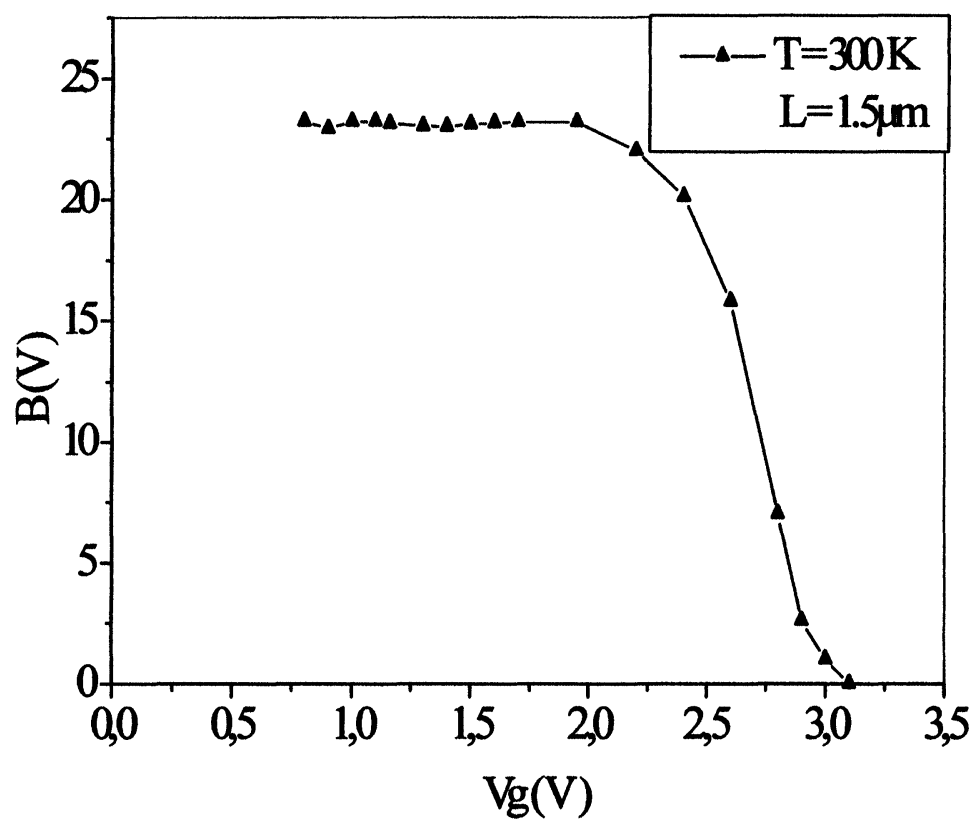

FIGURE $3 B$ parameter versus $V g$ characteristic for $1.5 \mu \mathrm{m}$ channel length at $T=300 \mathrm{~K}$.

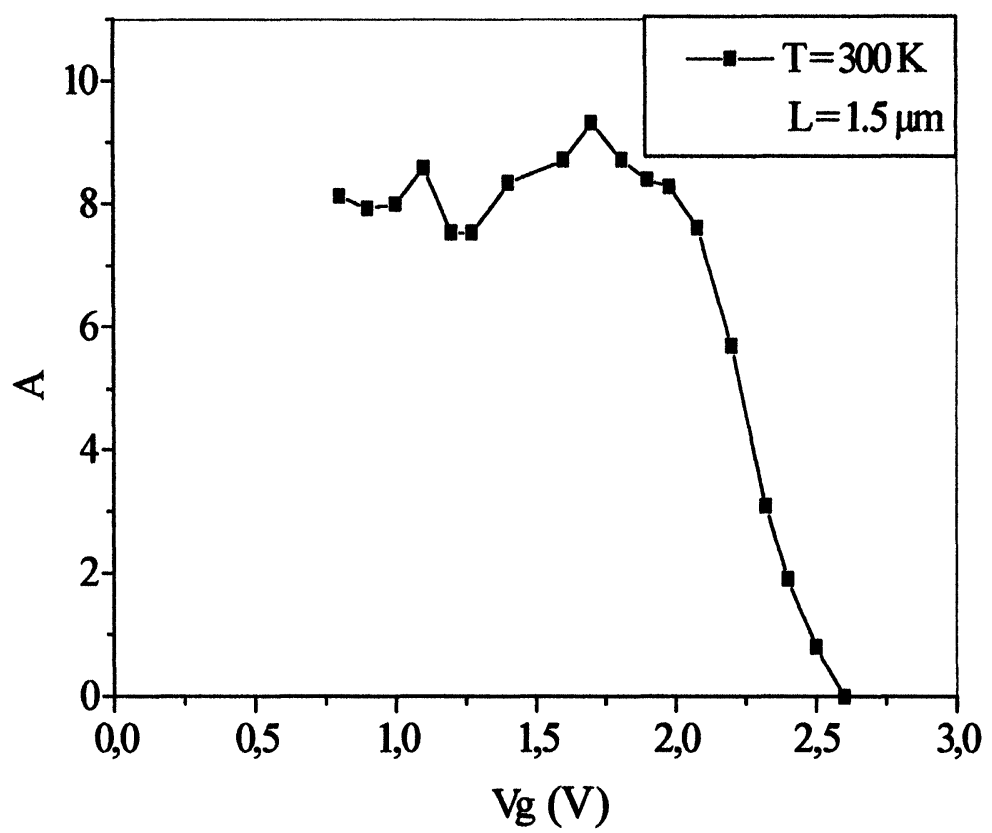

FIGURE 4 A parameter versus $V g$ characteristic for $1.5 \mu \mathrm{m}$ channel length at $T=300 \mathrm{~K}$. 


\section{CONCLUSION}

We conclude that this method is the alone method to find experimental continuous $V d_{\text {sat }}(V g)$ curves, and confirm in the same time the Isub/Id versus $\left(V d-V d_{\text {sat }}\right)$ relation. Its validity temperature range is found between liquid helium and room temperature. Its better applied in short channel MOSFET or in strong drain voltage for long channel MOSFET.

\section{References}

[1] Ghibaudo, G. (1987). "A simple Model for Drain Saturation Voltage with the Gate Voltage for short Channel MOSFETs”, Physica Status Solidi (a), 99, K149-K153.

[2] Jung, W.-Y., Wu, C.-Y. and Wu, H.-J. (1988). "A New Experimental Method to Determine the Saturation Voltage of Small Geometry MOSFETs", Solid State Electronics, 31(9), 1421-1431.

[3] Chan, T. Y., Ko, P. K. and Hu, C. (1984). "A Simple Method to Characterise Substrate Current In MOSFETs", IEEE Electron. Dev. Lett., 5, 505-507.

[4] Inewski, K. and Salama, C. T. (1991). "New Method for Extraction of Effective Channel Length In Submicron MOSFETs", Electronics Letters, 27(6), 508-509.

[5] Chynoweth, A. G. (1958). "Ionisation Rates for Electrons and Holes In Silicon", Physical Review, 109(5), 1537.

[6] Rais, K., Ghibaudo, G. and Balestra, F. (1993). "Temperature Dependence of the Substrate Current In Silicon CMOS Devices", Electronics Letters, 29(9), 778-780. 

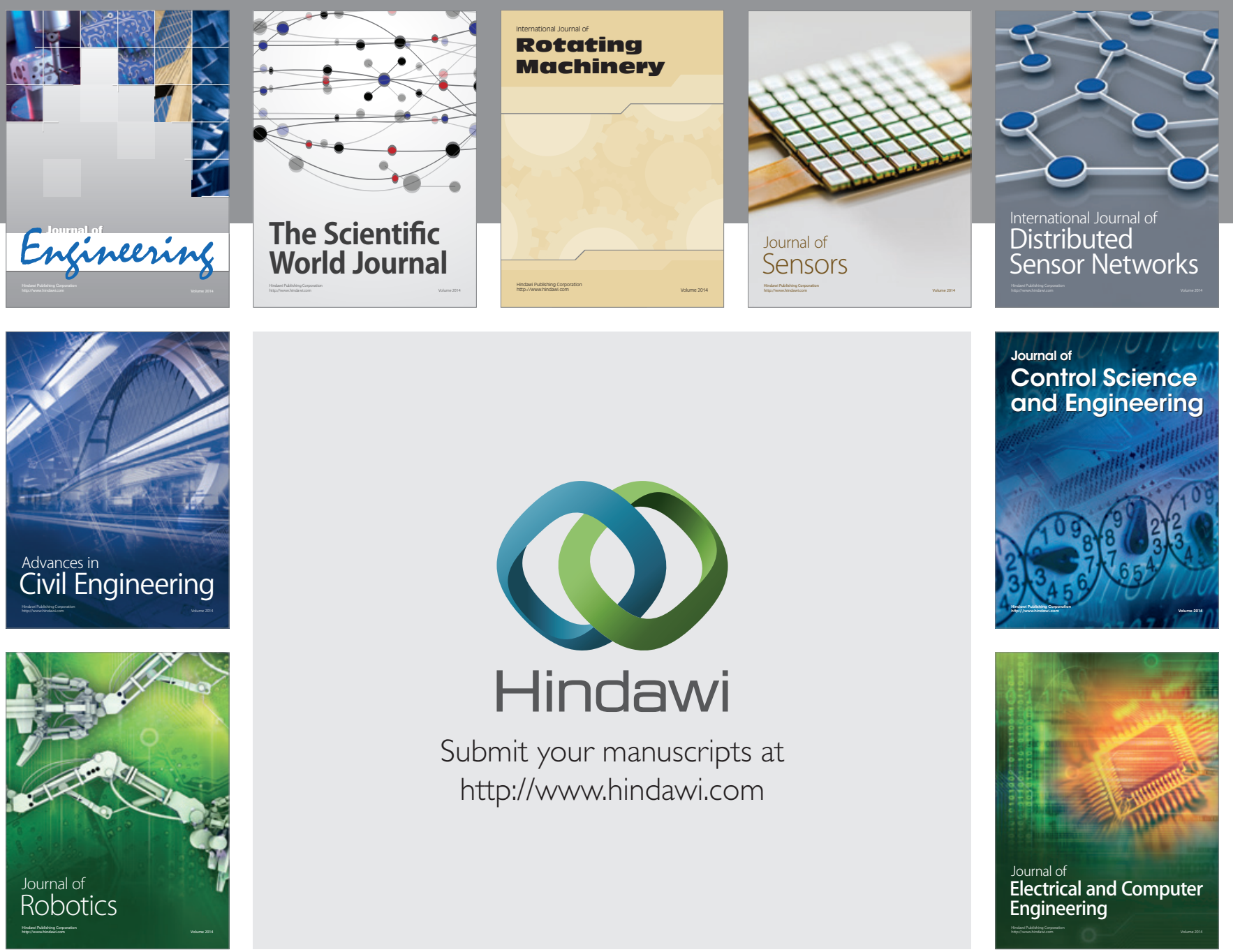

Submit your manuscripts at

http://www.hindawi.com
